\title{
Quality of Life for North Korean Female Refugees: The Influence of Physical Health, PTSD, and Social Support
}

\author{
Ji-yeon Lee \\ Hankuk University of Foreign Studies, Seoul, Korea \\ Sang-Soo Shin \\ Center for Mental Health, Melbourne School of Population and Global Health, \\ University of Melbourne \\ So Hee Lee \\ National Medical Center, Seoul, Korea
}

\begin{abstract}
Background of the Study. The purpose of this study was to test a mediation model that describes the pathways through which female North Korean defectors' perceived physical health and Post-traumatic stress disorder (PTSD) symptoms may be associated with their quality of life. Specifically, we aimed to investigate whether social support would mediate the association between North Korean defectors' perceived physical health and PTSD symptoms and their quality of life. Methods. The study sample included 172 female North Korean defectors living in South Korea. Participants completed a health condition checklist, the Posttraumatic Stress Disorder Checklist-Civilian Version (PLC-C), the Multidimensional Scale of Perceived Social Support (MSPSS), and the Korean version of the Short-Form 8-Item Health Survey (SF-8). Results. The structural equation model confirmed the hypothesized mediation model, with the following indices: $\chi^{2}(38)=86.184, \mathrm{CFI}=0.97, \mathrm{NFI}=$ $0.95, \mathrm{TLI}=0.96, \mathrm{RMSEA}=0.08$ (90\% CI: 0.06 - 0.10). Discussion. Our findings could help counselors understand unique issues that women refugees may experience as well as protective factors in their life (i.e., social support).
\end{abstract}

Keywords: Female North Korean defectors; physical health; post-traumatic stress disorder; quality of life; and social support

(c) 2019 Lee, Shin, \& Lee. Free to copy and share for education and scholarship under a Creative Commons Attribution NonCommercialNoDerivatives 4.0 License. 


\section{Background}

Severe political and economic conditions in North Korea have caused North Korean to defect to South Korea. The primary cause of defection in the 1990s was food shortages, but since the 2000s, the number of North Koreans who want to gain freedom and seek better opportunities for their children has increased. At present, approximately 33,000 people, which account for $7 \%$ of the total population of North Korea, have fled to S. Korea, and about $80 \%$ of North Korean defectors are women (Ministry of Unification, 2019). Although North Koreans have escaped from starvation and oppression, their quality of life (QOL) has become an important issue after their successful defection. Given the increasing number of defectors globally, understanding the factors associated with the QOL of North Korean defectors after resettlement can guide similar situations in other countries. This study aims to understand the unique issues encountered by female North Korean refugees who suffer from political and gender-based oppression. The results of this study can be useful in providing gender-appropriate care to female North Korean defectors.

QOL is important in the social adaptation process of North Korean defectors (Kwon, Lim, \& Kim, 2011). Numerous studies have focused on the effects of physical and psychological health on these defectors' QOL and adjustment in South Korea (Choi, Min, Cho, Joung, \& Park, 2011; Garuti \& Luerti, 2009; Hazell, Heaven, Kazemi, \& Fourie, 2009). North Korean defectors living in South Korea have reported poor perception of their physical health, thereby requiring extensive medical support (Korea Hana Foundation, 2014; Walen \& Lachman, 2000). North Korean defectors are exposed to a poor healthcare environment and are often unable to receive healthcare benefits even when they become long-term residents in China (Kang, 2018). Thus, particular attention should be provided to the physical health of women because North Korean female detectors often experience sexual assault or become victims of trafficking in the migration process (Kang, 2018). Before they come to South Korea, North Korean defectors spend an average of 5-7 years in China or other countries. Female North Korean defectors have reported lower health status compared with males (Wang, Yu, Noh, \& Kwon, 2014); thus, the physical health of these women should be examined to understand their QOL.

Apart from gynecological diseases (e.g., syphilis, cervical cancer, HIV infection), anxiety, and shock encountered during the migration process, North Korean female defectors have a high possibility of developing mental disorders, such as post-traumatic stress disorder (PTSD), which affect their QOL (Lee, 2006). Defectors tend to show signs of psychological and mental health concerns, such as symptoms of PTSD, depression, and anxiety (Goldney, Fisher, Wilson, \& Cheok, 2000). Among these concerns, PTSD is the most critical factor that influences defectors' mental health (Tyhurst, 1982). Trauma is also reported as a critical factor that undermines QOL (Araya, Chotai, Komproe, \& de Jong, 2007). According to previous studies, defectors who have experienced more prolonged asylum procedures report more severe PTSD than those who have experienced shorter procedures (Laban, Komproe, Gernaat, \& de Jong, 2008). Female North Korean defectors mainly reported higher rates of PTSD, depression (Kim, 2010), and anxiety (Bravell, Berg, Malmberg, \& Sundstrom, 2009) than their male counterparts. Traumatic events, such as social violence, cause serious trauma to women (Raphael, Taylor, \& McAndrew, 2008), and female North Korean defectors showed twice more PTSD symptoms than males (Kim \& Yoo, 2010). Thus, PTSD is one of the risk factors in the adjustment process, which may affect the QOL of female North Korean defectors.

Social support serves as a buffer against the physical and psychological effects of stress. According to the stress-buffering model by Cohen and Will (1985), social support helps individuals to deal with stress. Social support is considered a significant factor in enhancing the QOL of immigrant women and lowering suicide rates (Simich, Beiser, \& Mawani, 2003; Cho \& Haslam, 2010). Consequently, social support is one of the most important factors that influence the adjustment of North Korean defectors in South Korea (Kim, Lee, \& Kim, 2011). Social support facilitates the recovery from illness and surgical procedures (Kulik \& Mahler, 1989) and aids in the treatment of psychological injuries. Defectors may experience cultural adjustment similar to that of immigrants, while social support is a critical factor for a cross-cultural adjustment (Adelman, 1988). 
Thus, connections with the South Korean hosts would enable defectors to acquire the social and practical skills necessary to live in the country. In addition, acceptance by the North Korean community in South Korea can provide a sense of belonging (Park, Cho, \& Yoon, 2009). Previous empirical studies have shown that defectors' social relations can relieve and moderate life stress (Cobb, 1976) and influence QOL (Helgeson, 2003). QOL is negatively associated with difficulty in interacting with new people and communities, that is, with South Koreans in this case (Fitch, Bartholomew, Hanowski, \& Perez, 2015). In addition, North Korean defectors who live alone have experienced high levels of anxiety (Bravell et al., 2009), thereby demonstrating the importance of social support for the population. Empirical studies have emphasized that social support is vital for female North Korean defectors in South Korea by identifying the moderating effect of social support between their daily life stress and suicidal ideation (Kim, Choi, Chae, \& Hwang, 2013).

The Ministry of Unification of South Korea indicated that $80 \%$ of North Korean defectors who enter the country are women (Ministry of Unification, 2019). However, only a few studies have differentiated the experiences of female and male North Korean defectors. A few studies have shown that women are strongly affected by social networks, while others have determined that social support strongly affects the well-being of women (Walen \& Lachman, 2000). In a qualitative study on female North Korean adolescents living in South Korea, the gendered processes of escape, gender-discriminatory family culture, and vulnerable sexuality are identified as unique challenges for the population (Chung, Choi, \& Choi, 2013). These data are particularly relevant for female defectors who may have been exposed to sexual or domestic violence and gender discrimination during their escape; accordingly, social support is an important factor in recovering from the harmful effects of physical and mental health problems (Yap \& Devilly, 2004). Therefore, we analyzed social support as a mediating factor in the association between the physical and psychological health and QOL of female North Korean defectors.

From the psychology of liberation perspective, oppression deprives individuals and groups of their rights, whereas liberation promotes recovery (Prilleltensky, 2003). Liberation is the process of achieving psychological and political well-being, and for psychological well-being, the promotion of physical and mental health, and connections are needed (Prilleltensky, 2003). The liberation psychology theory has been used to promote social justice and understand refugees' socio-historical contexts of oppression. The current study focuses on the psychological well-being of female North Korean defectors. According to the liberation theory, at the relational level, welfare depends on cooperation and respect for diversity and social cohesion. For personal well-being, the promotion of physical and mental health is needed (Prilleltensky, 2003). Therefore, we considered physical and mental health as factors that influence female North Korean defectors' QOL at a personal level, and social support as factors to contribute to their QOL at the relational level.

The current study used the theoretical framework of liberation psychology and previous empirical studies related to female North Korean defectors as bases to test a hypothesized model of how physical and mental health (i.e., PTSD symptoms) would influence the QOL of female North Korean defectors. Moreover, this study tested such a model on how social support may have potential buffering effects of physical and psychological health on QOL.

\section{Method}

\section{Participants}

A total of $172(n=172)$ female North Korean defectors participated in the study. We excluded 2 participants who experienced difficulty answering questions due to severe mental health issues or dementia. Table 1 presents details of the demographic backgrounds of participants. 
Table 1. Participants' demographic backgrounds $(N=172)$

\begin{tabular}{llll} 
Variables & & Number & $\%$ \\
\hline Age, years & Range 19 - 76 & \\
& Mean $=47.45$ & \\
& $S D=10.61$ & & \\
Entry year & $2011-2013$ & 54 & 31.4 \\
& $2009-2010$ & 48 & 27.9 \\
& Before 2009 & 70 & 40.7 \\
Duration of stay in the third country & Straight to South Korea & 42 & 24.4 \\
& Under 5 years & 54 & 31.4 \\
& 5 years and more & 76 & 44.2 \\
Current employment & No & 118 & 68.6 \\
& Yes & 54 & 31.4 \\
Religion & & & 65.7 \\
Married & No & 113 & 34.3 \\
& Yes & 59 & 57.0 \\
Education in the North Korea & No & 98 & 43.0 \\
& Yes & 74 & 64.0 \\
\hline
\end{tabular}

Note. $S D=$ Standard deviation

\section{Procedures}

Participants were recruited from North Korean Defector Call Center and counseling centers caring for North Korean defectors with snowball sampling. The procedures of the study were thoroughly explained to all participants, and they signed written consent. All participants voluntarily completed self-report questionnaires on perceived physical health, PTSD, perceived social support, and QOL. The Institutional Review Board of the National Medical Center in South Korea approved the study. Structural equation modeling was used to test a mediation effect of social support in the association between the perceived physical health and PTSD symptoms and QOL.

\section{Measures}

\section{Demographic data}

We gathered information about the participants' entry year to South Korea, stay in the country, current occupation, income, religion, marital status, and education level, as experienced in North Korea.

\section{Perceived physical health}

To measure the perceived physical health status of North Korean refugees, we asked participants to describe their usual health condition and compared the answers with their health condition over the last year using a five-point Likert scale. Finally, we asked them to use a three-point Likert scale to compare their health condition with that of other people of the same age.

\section{Post-traumatic stress disorder}

The PTSD Checklist-Civilian Version (PCL-C), which consists of 17 items, was used to measure PTSD 
symptoms (Weisman, 1993). A five-point Likert scale from 1 ("not at all") to 5 ("extremely") was used to rate each PTSD symptom over the past month. A sample item included "Repeated, disturbing memories, thoughts, or images of a stressful experience?" The total score was used, with high PCL-C scores indicating a large number of PTSD symptoms. The measure was translated and the Korean version of the PCL-C showed good validity and reliability (Oh et al., 2014). In this sample, internal consistency was 0.97 .

\section{Perceived social support}

Social support was determined using the Multidimensional Scale of Perceived Social Support (MSPSS), which consists of 12 items with 3 subscales for family, friends, and significant others (Zimet, Powell, Farley, Werkman, \& Berkoff, 1990). A sample item included "There is a special person who is around when I am in need." We used the Korean version of the MSPSS in this study. The construct validity of the Korean version of the Multidimensional Scale of Perceived Social Support was tested previously on a population of Korean older adults (Kang et al., 2012). Items were measured on a five-point scale from 1 ("strongly disagree") to 5 ("strongly agree"). Total scores were used with high scores indicating high social support. Internal consistency was 0.95 in this sample.

\section{Quality of life}

The Short-Form 8-Item Health Survey (SF-8) was used to derive a health-related QOL (Ware, Kosinki, Dewey, \& Gandek, 2001; Han, Lee, Iwaya, Kataoka, \& Kohzuki, 2004). Two subscales include the physical health-related feature of QOL (PCS) and mental health-related features of QOL (MCS). A sample item included "During the fast 4 weeks, how much have you been bothered by emotional problems." The Korean version of the SF-8 was validated (Han, Lee, Kataoka, \& Kohzuki, 2004). In this study, the internal consistency of PCS was 0.88 , whereas that of MCS was 0.80 .

\section{Results}

Table 2. Zero-order Pearson Correlation among Variables $(N=172)$

\begin{tabular}{llllll} 
Variables & M & SD & $\mathbf{1}$ & $\mathbf{2}$ & $\mathbf{3}$ \\
\hline 1. Perceived Physical Health & 9.37 & 1.90 & - &. & \\
2. PTSD symptoms & 24.95 & 19.38 & $.35^{* *}$ & - & \\
3. Social Support & 41.09 & 12.31 & $-.29^{* *}$ & $-.53^{* *}$ & - \\
4. Quality of Life & 25.37 & 7.18 & $.60^{* *}$ & $.75^{* *}$ & $.44^{* *}$ \\
\hline
\end{tabular}

$p<.01^{\star *}$ Note. $M=$ Mean, $S D=$ Standard deviation

Data were normally distributed in univariate and multivariate analyses, thereby meeting structural equation modeling (SEM) assumptions. Table 2 presents the means and standard deviations of all variables, as well as their Pearson correlations. Multicollinearity was an unlikely problem considering low correlations at below 0.85 (Tabachnick \& Fidell, 2007).

We used SEM to test the hypotheses and then employed several fit indices to determine whether the hypothesized model was a good fit. The comparative fit index (CFI) indicates the relative fit between hypothesized and baseline models that assume no relationships among variables. The CFI ranges from 0 to 1.0 with values closer to 1.0 indicating a better fit. The normed fit index (NFI) is derived by comparing the hypothesis with the independence model. A value of 0.90 or above indicates a well-fitting model. The standardized RMSEA should be 0.05 or less in a well-fitting model. The proposed mediation model followed a two-step procedure. In the first step, confirmatory factor analysis (CFA) was conducted to develop a measurement model with an acceptable fit. Once the acceptable fit was established, the structural model was tested. The confirmatory model consisted of 4 latent and 11 observed variables. We used each item as an observed component of the latent variable because the measure of physical health only includes three items. For PTSD and QOL, we created item parcels using the 
item-to-construct balance method (Little, Cunningham, Shahar, \& Widaman, 2002). For social support, we used subscales as observed indicators of latent variables.

\section{Measurement model}

In the first SEM step, we tested the measurement model through CFA with the maximum likelihood estimation method using AMOS software. The measurement model showed good fit to the data with the following indices: $\chi^{2}(38)=86.18, \mathrm{CFI}=0.97, \mathrm{NFI}=0.95$, TLI $=0.96$, and RMSEA $=0.08$ (90\% CI: 0.06-0.10). All factor loadings were significant $(\mathrm{p}<0.001)$, indicating that the latent variables were well represented by the indicators.

\section{Structural model}

\section{Fig. 1. The Hypothesized Structural Model}

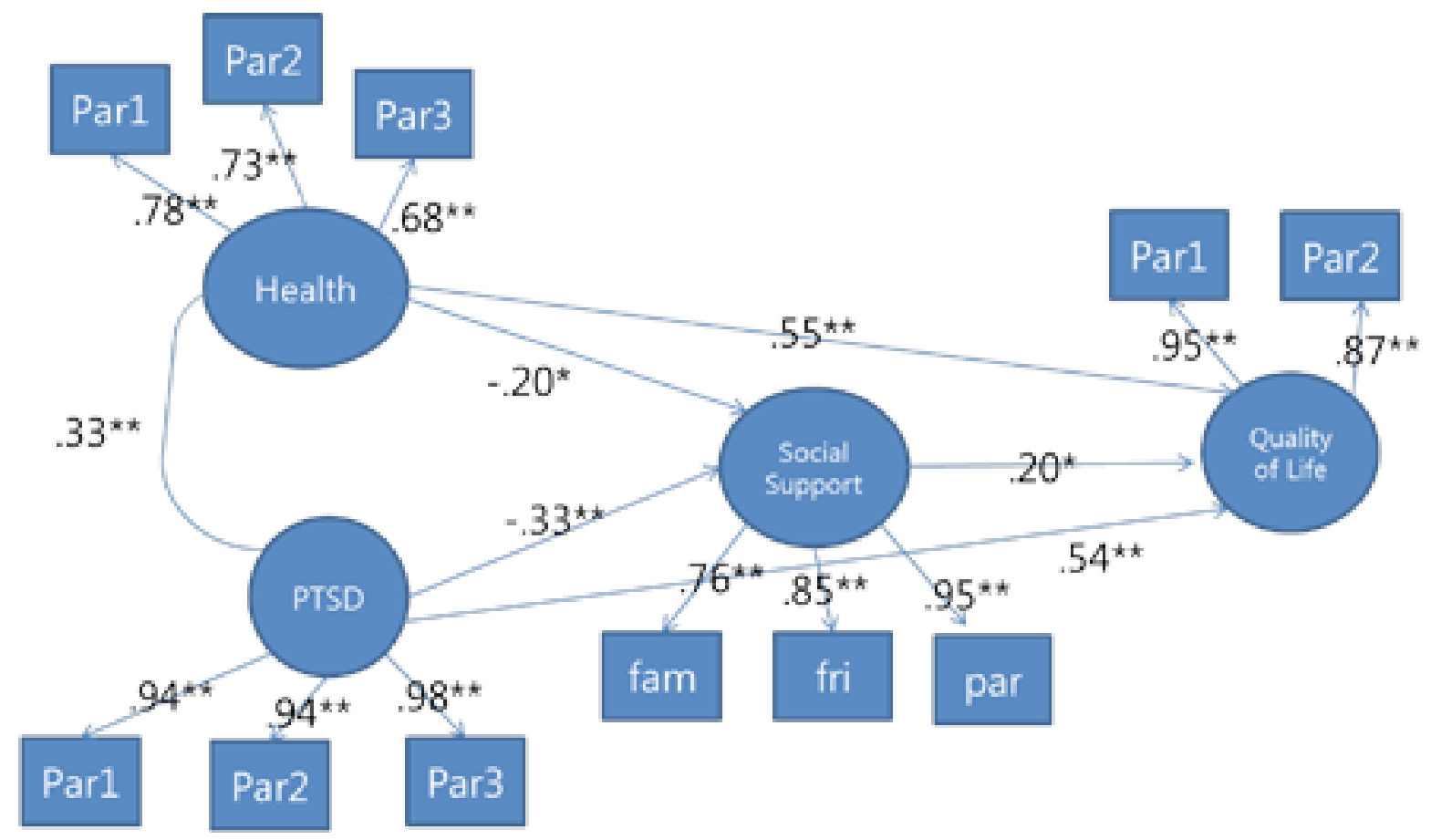

${ }^{\star} p<.05 .{ }^{* *} p<.01$

The hypothesized structural model (see Figure 1) was evaluated to identify the mediating relationships among the variables in the hypothesized model. The structural model provided good fit to the data: $\chi^{2}(38)=$ 86.184, $\mathrm{CFI}=0.97, \mathrm{NFI}=0.95, \mathrm{TLI}=0.96$, and $\mathrm{RMSEA}=0.08(90 \% \mathrm{CI}: 0.06-0.10)$. 
Table 3. Goodness-of-Fit Indices for Model Testing

\begin{tabular}{llllllll} 
& $\chi^{2}$ & df & $\Delta \chi^{2}$ & $\Delta$ df & CFI & TLI & \\
\hline Hypothesized Model & 86.18 & 38 & & & .97 & .96 & .08 \\
Alternative Model 2A & 146.83 & 39 & 60.65 & 1 & .94 & .91 & .12 \\
Alternative Model 2B & 111.01 & 39 & 24.83 & 1 & .96 & .94 & .10 \\
Alternative Model 2C & 285.65 & 40 & 199.47 & 2 & .86 & .81 & .18 \\
\hline
\end{tabular}

Note. Alternative Model 2A is a model without a direct path between physical health and quality of life, Alternative Model 2B is a model without a direct path between PTSD and quality of life, Alternative Model 2C is a model without direct paths between both physical health and PTSD to quality of life.

$\Delta \chi^{2}=$ Chi-squared difference test between the Hypothesized Model and the Alternative Models, $\mathrm{CFI}=$ Comparative Fit Index, TLI = Tucker Lewis Index, RMSEA = Root Mean Square Error of Approximation

To determine the presence of full or partial mediation, we compared the proposed model with a model without a direct path between perceived physical health and QOL (alternative model 2A), PTSD symptoms, and QOL (alternative model 2B), and perceived physical health and PTSD symptoms to QOL (alternative model 2C). The fit values of the three alternative models were significantly lower than those of the hypothesized model. Alternative models 2A, 2B, and 2C produced lower CFI, TLI, RMESA, and chi-square values compared with the hypothesized model. Chi-square difference tests between the hypothesized and alternative models indicated that the hypothesized model yielded a significantly better fit to the data compared with any of the alternative models. A difference of $\Delta \chi^{2}=60.65, \Delta \mathrm{df}=1$ was found between the hypothesized and alternative model $2 \mathrm{~A}$, $\Delta \chi^{2}=24.83, \Delta \mathrm{df}=1$ between the hypothesized and alternative model $2 \mathrm{~B}$, and $\Delta \mathrm{\chi}^{2}=199.47, \Delta \mathrm{df}=2$ between the hypothesized and alternative model $2 \mathrm{C}$. Therefore, the hypothesized full mediation model resulted in a better fit to the data than any of the alternative models (see Table 3).

\section{Discussion}

This study investigated the association between the perceived physical health and PTSD symptoms and the QOL of female North Korean defectors living in South Korea. The current research also tested the mediating effects of social support related to this association. Previous studies have determined that physical and mental health conditions (e.g., PTSD symptoms) are risk factors that diminish the QOL. By contrast, social support can be a protective factor. The results of this study are consistent with those of previous research and supported that PTSD is negatively associated with the QOL of North Korean defectors (Helgeson, 2003). The current study also confirmed that physical health influences their QOL and social adjustment (Choi, Min, Cho, Joung, \& Park, 2011; Garuti \& Luerti, 2009; Hazell, Heaven, Kazemi, \& Fourie, 2009). Furthermore, the present research is consistent with previous studies, which have indicated that North Korean defectors who escaped without families tended to be depressed (Jeon et al., 2009), while social support is associated with improved QOL (Kato et al., 2013).

The focus on female North Korean defectors and the role of social support in mediating the negative impact of their perceived health status and PTSD symptoms on their QOL is the unique feature and contribution of this study. Several studies have addressed the physical and mental health status of North Korean defectors and their QOL (Choi et al., 2011; Garuti \& Luerti, 2009). One study has tested the factors associated with the physical health of North Korean defectors who have reported poor health status (Wang, Yu, Noh, \& Kwon, 
2014). Prior studies have shown that female North Korean defectors have higher prevalence rates (29.5\%) of PTSD than their male counterparts (Jeon et al., 2005). Female North Korean defectors experience unique issues, such as the gendered process of escape, gender-discriminatory family culture, and vulnerability to sexual assault (Mitus \& Coughlin, 2013). However, no study has specifically explored pathways of the mental and physical health and QOL of female North Korean refugees. The analysis of gender-specific factors that influence female North Korean defectors' QOL is important because of their high rates of psychological symptoms and poor health status.

We considered social support as a mediating factor in the association between physical health and PTSD and QOL. Previous studies have indicated that North Korean defectors living with their spouses have better QOL than those who do not (Bravell et al., 2009). However, prior studies have also indicated that couples often have conflicts once they settle in South Korea because women adapt relatively faster than men, thereby altering the couple's roles and positions (Choi, 2011). The difficulty experienced by North Korean defectors in getting along with South Korean citizens may reduce their QOL by decreasing opportunities to learn the social norms of a new culture and undermining the feeling of belonging. A study on the association between defense mechanisms and PTSD symptoms among North Korean defectors has indicated that social isolation is associated with undoing and isolation (Jun et al., 2015). Thus, North Korean defectors may use interpersonal isolation as a maladaptive coping strategy. Therefore, practitioners who work with female North Korean defectors can help improve their QOL by helping them identify ways to gain social support in their given social context (e.g., by facilitating social skills training and exposing them to South Korean popular culture). Apart from general and practical settlement training, interpersonal training would be beneficial to reduce difficulties in everyday communication. Compared with perceived physical health and PTSD symptoms, changing the level of social support that female North Korean defectors seek and receive may be substantially easy for clinicians. Researchers and practitioners who work with female defectors can help enhance their QOL by helping them gain social support, thereby serving as a buffer against the negative effects of physical and mental health on their QOL.

Difficulties in healthcare access are common problems for defectors or immigrants (Topa, Neves, \& Nogueira, 2013). These difficulties are intensified in the sexual and reproductive health domains for female defectors (Machado et al., 2009). Therefore, female defectors should be assisted to have access to the needed health care. From the theoretical perspective of migration, in which immigration causes stresses and health problems (Im \& Yang, 2006), such protective factors as social support can be beneficial. The acculturation process also facilitates the healthcare-seeking behaviors of immigrants (Im \& Yang, 2006), while social support can accelerate their acculturation process. The results of the study align with the liberation psychology movement of engaging with the marginalized population (Burton \& Kagan, 2004), where the connection is needed for the liberation of refugees.

The physical and mental health status of female North Korean defectors can be understood as social conditions associated with oppression at a macro level, while limited social support can be understood as socialbased oppression at the micro-level (Bartky, 1990). Based on this, the research results have two-level action plans; one at the system level and another at the interpersonal level. For the system level, the results of this study suggest the importance of a gender-sensitive system that would require the support of the Office of Women's Policy under the Ministry of Unification. Gender-specific barriers and protective factors must be identified to develop gender-sensitive programs for North Korean defectors. The current research is one of the few empirical studies that have analyzed the gender-specific barriers and protective factors for female North Korean defectors. At the interpersonal level, we need to view social support from a broad perspective. Accordingly, collective actions are needed to foster bond with one's reference group and bridge across other groups (Moane, 2003; Putnam, 2000). The current study focuses on the psychological well-being of female North Korean defectors, and we considered their physical and mental health, and social support as factors to contribute to their QOL as 
a part of the liberation process.

\section{Global Implications in Counseling Psychology}

Since the presidential address at the Society of Counseling Psychology Division 17 in 2003, the globalization of counseling psychology has been illuminated. Increased attention has focused on women as victims of human rights violations at the international level. According to the World Health Organization reports, women experience much more psychological distress than men. Furthermore, the feminist perspective has relevance for all women across borders, and clinicians need to consider the social contexts of women's psychological distress. With globalization, counselors need to know how to work with women from an international background and become familiar with their unique challenges. Even in the United States, 1 in 10 people is an immigrant or has a refugee background (US Census Bureau, 2010). The results of this study would help counselors to understand unique issues that women refugees may experience as well as a protective factor in their QOL (i.e., social support).

\section{Limitation}

Several limitations should be considered when evaluating the results of this study. First, the self-report nature of the instruments used limits the researchers' ability to determine how truthfully the respondents answered. Second, this study may not be generalizable to all female North Korean defectors in South Korea because the current sample fails to represent their entire population. Lastly, this study measured the perceived physical health of North Korean defectors instead of their actual physical health status. Thus, future studies should analyze the effects of actual physical health or differentiate the effects of perceived physical health and actual physical health status.

\section{Acknowledgment}

This study was supported by a grant from the Korean Mental Health Technology R\&D Project, Ministry of Health \& Welfare, Republic of Korea (HL19C0007). Ji-yeon Lee's work was supported by the Research Fund of Hankuk University of Foreign Studies.

\section{Declaration of Conflict of Interest}

The authors declare no conflict of interest with respect to the research, authorship, and/or publication of this article.

\section{Corresponding Author}

So Hee Lee, MD, PhD. Department of Psychiatry, National Medical Center, 245, Eulji-ro, Jung-gu, Seoul, 04564, Rep. of KOREA. Tel +82-2-2260-7311, Fax +82-2-2268-5028, E-mail psyhee@hanmail.net 


\section{References}

Adelman, M. B. (1988). Cross-cultural adjustment: A theoretical perspective on social support. International Journal of Intercultural Relations, 12(3), 183-204.

Araya, M., Chotai, J., Komproe, I. H., \& de Jong, J. T. (2007). Effect of trauma on quality of life as mediated by mental distress and moderated by coping and social support among postconflict displaced Ethiopians. Quality of Life Research, 16(6), 915-927. doi:10.1007/s11136-007-9201-9

Bartky, S. L. (1990). Femininity and domination. Studies in the phenomenology of oppression. London: Routledge.

Bravell, M. E., Berg, S., Malmberg, B., \& Sundström, G. (2009). Sooner or later? A study of institutionalization in late life. Aging Clinical and Experimental Research, 21(4-5), 329-337.

Burton, M., \& Kagan, C. (2004). Liberation psocial psychology: Learning from Latin America, Journal of Community and Applied Social Psychology, 15, 63-78.

Cho, Y. B., \& Haslam, N. (2010). Suicidal ideation and distress among immigrant adolescents: The role of acculturation, life stress, and social support. Journal of Youth and Adolescence, 39(4), 370-379.

Choi, S. K. (2011). A Study on the Issues of North Korean Female Defectors' Adaptation to South Korean Society from Gender-cognitive Perspective and the Measures to Support their Settlement. The Korean Association of Peace Studies, 12(4), 327-348.

Choi, S. K., Min, S. J., Cho, M. S., Joung, H., \& Park, S. M. (2011). Anxiety and depression among North Korean young defectors in South Korea and their association with health-related quality of life. Yonsei Medical Journal, 52(3), 502-509. doi:10.3349/ymj.2011.52.3.502

Chung, H. S., Choi, Y. H., \& Choi, J. E. (2013). Factors impeding the successful settlement of female adolescent immigrants from North Korea and measures to support them. Korean Women's Development Institute, 6, 104-125.

Cobb, S. (1976). Social support as a moderator of life stress. Psychosomatic Medicine, 38(5), 300-314.

Cohen, S., \& Wills, T. A. (1985). Stress, social support, and the buffering hypothesis. Psychological Bulletin, 98(2), 310.

Fitch, G. M., Bartholomew, P. R., Hanowski, R. J., \& Perez, M. A. (2015). Drivers' visual behavior when using handheld and hands-free cell phones. Journal of Safety Research, 54, 105-e29.

Garuti, G., \& Luerti, M. (2009). Hysteroscopic bipolar surgery: a valuable progress or a technique under investigation?. Current Opinion in Obstetrics and Gynecology, 21(4), 329-334.

Goldney, R. D., Fisher, L. J., Wilson, D. H., \& Cheok, F. (2000). Major depression and its associated morbidity and quality of life in a random, representative Australian community sample. Australian and New Zealand Journal of Psychiatry, 34(6), 1022-1029.

Han, C. W., Lee, E. J., Iwaya, T., Kataoka, H., \& Kohzuki, M. (2004). Development of the Korean version of Short-Form 36-Item Health Survey: health related QOL of healthy elderly people and elderly patients in Korea. The Tohoku journal of experimental medicine, 203(3), 189-194.

Hazell, W., Heaven, D., Kazemi, A., \& Fourie, D. (2009). Atrio-oesophageal fistula: An emergent complication of radiofrequency ablation. Emergency Medicine Australasia, 21(4), 329-332.

Helgeson, V. S. (2003). Social support and quality of life. Quality of Life Research, 12 Suppl 1, 25-31.

Im, E. O., \& Yang, K. (2006). Theories on immigrant women's health. Health Care for Women International, $27(8), 666-681$. 
Prilleltensky, I. (2003). Understanding, resisting, and overcoming oppression: Toward psychopolitical validity. American Journal of Community Psychology, 31(1-2), 195-201.

Jeon, B. H., Kim, M. D., Hong, S. C., Kim, N. R., Lee, C. I., Kwak, Y. S., . . Kim, D. S. (2009). Prevalence and Correlates of Depressive Symptoms among North Korean Defectors Living in South Korea for More than One Year. Psychiatry Investigation, 6(3), 122-130. doi:10.4306/pi.2009.6.3.122

Jeon, W., Hong, C., Lee, C., Kim, D. K., Han, M., \& Min, S. (2005). Correlation between traumatic events and posttraumatic stress disorder among North Korean defectors in South Korea. Journal of Traumatic Stress, 18(2), 147-154. doi:10.1002/jts.20017

Jun, J. Y., Lee, Y. J., Lee, S. H., Yoo, S. Y., Song, J., \& Kim, S. J. (2015). Association between defense mechanisms and psychiatric symptoms in North Korean Refugees. Comprehensive Psychiatry, 56, 179-187. doi:10.1016/j.comppsych.2014.10.001

Kang, D. Y. (2018). Social Adjustment of North Korean Defectors and Support Plans for Their Settlement. The Journal of Political Science and Communication, 21(2), 185-205.

Kang Y. H., Kim M. Y., Lee G. J., Jung D. Y., \& Ma R. W. (2012). A study of social support, loneliness, sleep quality, and perceived health status among community-dwelling older adults. Journal of Korean Public Health Nursing, 26(2), 303-313.

Kim, B., \& Yoo, S. (2010). North Korean Defectors Panel Study: Economic Adaptation, Mental Health Physical Health. Seoul, Korea: North Korean Refugees Foundation.

Kim, H. A., Lee, S. Y., \& Kim, K. S. (2011). The Relationship between Resiliance and Subjective Well-Being: Based on Korean Immigrants and North Korean Refugees. Korean Journal of Counseling, 12(1), 333354.

Kim, H. K. (2010). The relations between defense mechanism and mental health problems of North Korean Female Refugees. The Korean Journal of Woman Psychology, 15(1)155-173.

Kim, J. Y., Choi, K. H., Chae, J. H., \& Hwang, H. J. (2013). Life Stress, Social Support and Suicidal Ideation of North Korean Refugee Women in South Korea. Korean Journal of Social Welfare Studies, 44(2), 35-58.

Korea Hana Foundation(2014). Survey of North Korean defectors living in South Korea. Seoul, Korea Hana Foundation. Retrieved from http://www.dbpia.co.kr/SKnowledge/ArticleDetail/.

Kulik, J. A., \& Mahler, H. I. (1989). Social support and recovery from surgery. Health Psychology, 8(2), 221238.

Kwon, K. D., Lim, J. B., \& Kim, D. J. (2011). The impact of the quality of life in North Korean settlers. Journal of the Korean Association for Local Government Studies, 23, 207-230.

Laban, C. J., Komproe, I. H., Gernaat, H. B., \& de Jong, J. T. (2008). The impact of a long asylum procedure on quality of life, disability and physical health in Iraqi asylum seekers in the Netherlands. Social Psychiatry and Psychiatric Epidemiology, 43(7), 507-515. doi:10.1007/s00127-008-0333-1

Lee, K. S. (2006). A Study on the Social Adaptation Process of Women Migrants. The Journal of Asian Women, 45(1), 191-234.

Little, T. D., Cunningham, W. A., Shahar, G., \& Widaman, K. F. (2002). To parcel or not to parcel: exploring the question, weighing the merits. Structural Equation Modeling, 9, 151-173.

Machado, M. D. C., Fernandes, A., Padilla, B., Dias, S., Gomes, I., Dias, A., \& Oliveira da Silva, M. (2009). Maternal and child healthcare for immigrant populations. Bruxelas: International Organization for Migration. 
Ministry of Unification. (2019). North Korean defector policy administrative data and statistics. Seoul: Ministry of Unification. Retrieved from https://www.unikorea.go.kr/unikorea/business/statistics/

Mitus, A. J., \& Coughlin, L. (2013). The Value of Actionable Content in a Clinical Setting: Access to Better Information Facilitates Enhanced Cancer Care. American Health \& Drug Benefits, 6(2), 104.

Moane, G. (2003). Bridging the personal and the political: Practices for a liberation psychology, American Journal of Community Psychology, 31, 91-101.

Oh, S.I., Won, S. D., Lee, S. H., Yoo, S. Y., Kim, H.C., \& Kim, H. J. (2014). Reliability and validity of the Korean version of the PTSD checklist civilian version in North Korean Defectors, . Journal of Korean Neuropsychiatry Association, 53(6), 410-417.

Park, K., Cho, Y., \& Yoon, I. J. (2009). Social inclusion and length of stay as determinants of health among North Korean refugees in South Korea. International Journal of Public Health, 54(3), 175-182. doi:10.1007/s00038-009-8048-y

Putnam, R. D. (2000). Bowling alone: America's declining social capital. In Culture and politics (pp. 223-234). Palgrave Macmillan, New York.

Raphael, B., Taylor, M., \& McAndrew, V. (2008). Women, catastrophe and mental health. Australian and New Zealand Journal of Psychiatry, 42(1), 13-23.

Simich, L., Beiser, M., \& Mawani, F. N. (2003). Social support and the significance of shared experience in refugee migration and resettlement. Western Journal of Nursing Research, 25(7), 872-891.

Tabachnick, B. G., \& Fidell, L. S. (2007). Experimental Designs Using ANOVA. $1^{\text {st }}$ Edition. Belmont(CA), Thomson/Brooks/Cole.

Topa, J., Neves, S., \& Nogueira, C. (2013). Immigration and health: women immigrants'(in) ability to access health care. Saúde e Sociedade, 22(2), 328-341.

Tyhurst, L. (1982). Coping with refugees. A Canadian experience: 1948-1981. International Journal of Socical Psychiatry, 28(2), 105-109.

Walen, H. R., \& Lachman, M. E. (2000). Social support and strain from partner, family, and friends: Costs and benefits for men and women in adulthood, Journal of Social and Personal Relationships, 17, 5-30

Wang, B. R., Yu, S., Noh, J. W., \& Kwon, Y. D. (2014). Factors associated with self-rated health among North Korean defectors residing in South Korea. BMC Public Health, 14, 999. doi:10.1186/1471-2458-14-999

Ware, J. E., Kosinski, M., Dewey, J. E., \& Gandek, B. (2001). How to score and interpret single-item health status measures: a manual for users of the SF-8 health survey. Lincoln, RI: QualityMetric Incorporated, 15(10), 5 .

Weisman, G. K. (1993). Adolescent PTSD and developmental consequences of crack dealing. American Journal of Orthopsychiatry, 63(4), 553-561.

Wilson, W. J. (2011). When work disappears: The world of the new urban poor. Vintage.

Zimet, G. D., Powell, S. S., Farley, G. K., Werkman, S., \& Berkoff, K. A. (1990). Psychometric characteristics of the Multidimensional Scale of Perceived Social Support. J Pers Assess, 55(3-4), 610-617. doi:10.1080/00 223891.1990.9674095

Yap, M. B., \& Devilly, G. J. (2004). The role of perceived social support in crime victimization. Clinical Psychology Review, 24(1), 1-14. 